\title{
Hesitation in Intercultural Communication: Some Observations and Analyses on Interpreting Shoulder Shrugging
}

\author{
Kristiina Jokinen ${ }^{1}$ and Jens Allwood ${ }^{2}$ \\ ${ }^{1}$ Department of Speech Sciences, University of Helsinki \\ PO Box 9, 00014 University of Helsinki, Finland \\ Kristiina.Jokinen@helsinki.fi \\ ${ }^{2}$ SSKKII, University of Göteborg \\ Box 200, 40530 Göteborg \\ jensaling.gu.se
}

\begin{abstract}
This paper concerns the different ways in which hesitation, and hesitation related phenomena like uncertainty, doubt and other phenomena where lack of knowledge is involved are expressed in different cultures. The paper focuses especially on shoulder shrugging as a signal of hesitation or uncertainty, and starts from the observation that shoulder shrugging has different interpretations depending on the interlocutor's cultural background. It is not commonly used in Eastern cultures while in Western cultures it is a sign of uncertainty and ignorance. The paper reports a small study on the differences in interpretation of a particular video tape gesture, and draws some preliminary conclusions of how this affects intercultural communication between human interlocutors and between humans and conversational agents.
\end{abstract}

Keywords: hesitation signalling, gesturing, intercultural communication.

\section{Introduction}

Intercultural communication (ICC) is usually defined as communication between people who do not have the same ethnic or national cultural background, e.g. communication between a Chinese person and a German person (cf. [5], [1]). One of the goals of the study of ICC is to discover similarities and differences in the way people from different cultures communicate through cross-cultural comparative studies. With this cross-cultural information as a background, it is then possible to study whether and how such differences influence intercultural communication between people who have different cultural backgrounds.

There are many types of studies of intercultural communication. The most common type is questionnaire based cross-cultural studies of differences in attitudes and values. Probably the most well known is the IBM-study reported by [5]. Another approach is to observe people's communicative behaviour, based on audio or video recordings of actual interactions in different cultures (leading to cross-cultural comparison) or on recordings of intercultural interaction. This line of research ranges 
from linguistic-cultural studies to computational modelling of cultural behaviour patterns and has become more common with video corpora being collected and easily available for analysis. Usually, there are three different possible foci for such studies: (i) differences in produced communicative behaviour, (ii) differences in interpretation and understanding of the behaviour produced and (iii) studies of how context influences the communicative behaviour produced or its interpretation. For instance, [9] studied communicative behaviour of Finns and Estonians, and found differences in speaking rate, length of pauses and turns, and interruptions. As she discussed the differences in terms of high-context and low-context cultures ([4]), she concluded that the communication even among people from neighbouring countries with closely related languages like Finnish and Estonian can have differences that reflect cultural differences: Estonian speakers show higher speech tempo, short pauses, and frequent interruptions of interlocutors, which are claimed to be features of low-context cultures, whereas Finnish speakers exhibit opposite features typical to high-context cultures. [8], similarly, studied Finnish and English communication strategies from the point of view of shared context and common ground, and noticed that misinterpretations in various everyday dialogue situations depend on cultural presuppositions of the interlocutors. This can be related to the continuum of highcontext vs. low-context cultures. They also pointed out that dialogue strategies are learnt through interaction, they also involve presupposition, and that awareness of cultural differences can help in avoiding miscommunication in ordinary activities such as everyday conversations. In virtual agent technology, cultural differences have been actively studied so as to produce appropriate behaviour in virtual conversational agents, and computational models for culture specific communicative behaviour of such agents have recently been built, see e.g. [6] and [3].

The three main foci of intercultural studies may be further subdivided in several ways. For instance, the produced communicative behaviour may be divided in (i) vocal (verbal and non-verbal), (ii) written (verbal and non-verbal e.g. pictures) and (iii) body movements (verbal and non-verbal), depending on the medium in which the communicative context is mediated. Interpretation may be subdivided in factual and emotional interpretation and context can be subdivided into, for example, physical setting and social setting. In this paper we are interested in body movement (shoulder shrug) and its factual interpretation in a social context, and we assume that the type of social setting is influenced by the social activity in which the interlocutors are involved in.

The goal of this paper is to study the different ways in which phenomena like hesitation, uncertainty, doubt and other phenomena where lack of knowledge is involved are expressed in different cultures. We will refer to this as "hesitation related phenomena". We are also interested in how a given communicative behaviour - the shoulder shrug (which in many Western cultures is used to express lack of knowledge) - is interpreted by people with different cultural backgrounds. This research is part of an on-going collaborative project between three universities in the Nordic countries (Sweden, Finland, Denmark), which also includes a larger intercultural focus on bodily communication in different cultural settings (see more information on the NOMCO-project at the project website: http://sskkii.gu.se/nomco).

In general, hesitation related phenomena can be expressed in a number of different ways: 
1. Facial expression

2. Head movement

3. Shoulder movement

4. Prosody (e.g. lengthening or pausing)

5. Special words like $e h$ or $h m$

In the annotations of conversational speech, such phenomena as fillers (e.g. filled pauses, discourse markers, editing terms, and parentheticals) and disfluencies are also considered as hesitation markers, as well as self-corrections (repairs) and retractions (reformulation or restart of one's utterance). Many of these phenomena have been grouped under the heading "Own Communication Management" (OCM), see [2], referring to their function when they are often simultaneously used to hold a turn and to gain time for the speaker to choose how to continue speaking.

Some of the ways used to express hesitation seem very wide spread, some perhaps even universal, while others are more specific to certain cultures. If we consider the shoulder shrug, we can, for example, hypothesize that the following three functions are good candidates for being universal; a shoulder shrug is produced, because of an itch, a muscular discomfort, or psychological tension (e.g. a so called tick). The interpretation of a shoulder shrug as lack of knowledge is, however, more uncertain and is what this article represents an attempt to study.

In order to study the connection between shoulder shrugging and hesitation in a more systematic way, especially the interpretation of shrugging as a sign of uncertainty, we conducted a small empirical study using our recorded video data. We showed a video of a shoulder shrug to people of different cultural background, and asked them to interpret the gesture and tell us about their own use of a similar gesturing. The main purpose was to get data on two aspects related to body movements as communicative signals: do people notice certain kinds of body movements, and how do they interpret them in the communicative context.

The article discusses the study and its findings, and is structured as follows. We will present the video data and the setup for the questionnaire and interview study conducted in Japan and Sweden in Section 2. In Section 3, we will first present the results and observations, and then continue with the discussion of their interpretation and significance in general. We will conclude and point to some future research lines in Section 4.

\section{Data}

\subsection{Video Clip}

The particular shoulder shrug that prompted the study occurs in a video clip that is part of the dialogue data collected under the auspices of ATR/NICT in Japan [7]. The data contains three free-flowing conversations among four participants and each conversation is about 1,5 hours long. One of the participants is Japanese, while the other three come from three different countries in Europe but are familiar with Japanese culture due to living or having lived in the country. The dialogues are conducted in English, and topics vary from casual chatting and story telling to travel information and cultural conventions. 
The particular clip that is the focus of interest in this paper is part of a long discussion concerning how to address people in Japanese and in other languages, and how foreigners and people who do not master the language well usually make mistakes concerning the politeness code of each culture. The person using a shoulder shrug has been explaining the use of the French pronouns vous and $t u$, and how foreigners sometimes use the incorrect form. The speaker also uses his hand, which he first keeps close to his mouth but then stretches it towards the partners simultaneously extending his index finger and opening up his palm slightly. He then draws his hand back towards the mouth, and keeps his position during the clarification question by partner B, and finally shrugs at the end. The silenced finish of his sentence is then picked up by partner $\mathrm{C}$ who offers an explanation: "you get quite a lot of things excused if you're a foreigner". This prompts partner D to continue her earlier story about how to help foreigners to address Japanese people appropriately, while the partner B replies to this by taking it as a basis for his humorous utterance. This makes all the participants laugh, and thus functions as a release of the slightly embarrassed and tensed situation. The conversation then continues in a different mode, with partner $\mathrm{C}$ initiating a new although related topic.

The transcript of the pertinent part of the conversations is given below, and Figures 1 and 2 (next page) present snap-shots of the relevant shoulder shrug from sideways and from the front, by two cameras.

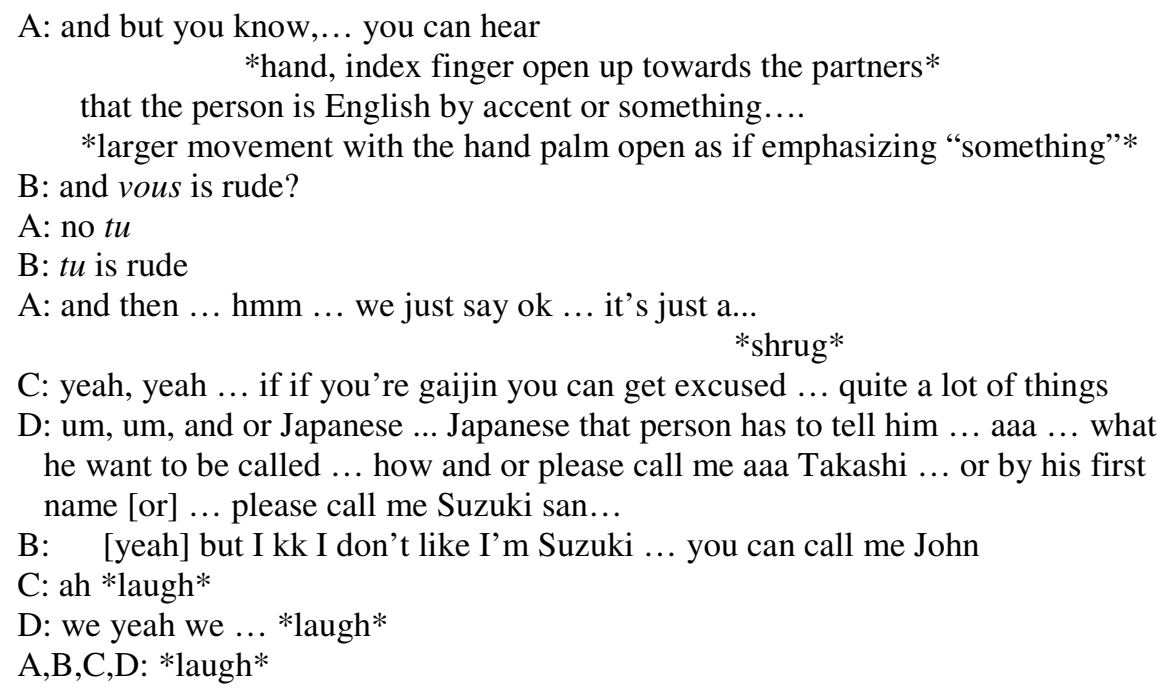

Most people within Western cultures interpret the shrug as a sign of hesitation or uncertainty, as if the person does not really know how to express oneself or continue the sentence, but this seems not to be so for everyone in Eastern cultures. A Japanese annotator, for instance, noticed the shrug as a peculiar gesturing which is not common in Japanese culture: in fact, shrugging of one's shoulders is seldom used on any occasion. Instead, the common way of expressing hesitation in Japan instead is to tilt one's head sideways and to prolong the words in the utterance so desu nee (roughly translated as "well it is so"). This also seems to be the case with other Eastern cultures as confirmed by our interview and questionnaire study. 

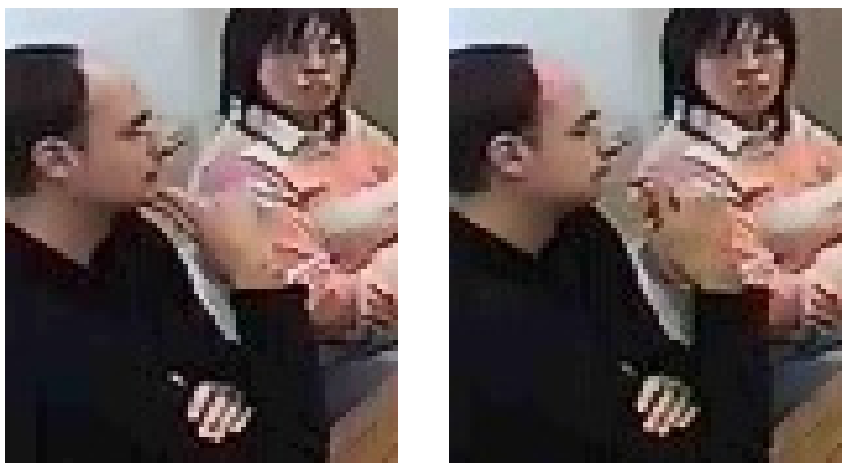

Fig. 1. Snapshot of the interlocutor on the front left shrugging his shoulders after uttering it's just a....
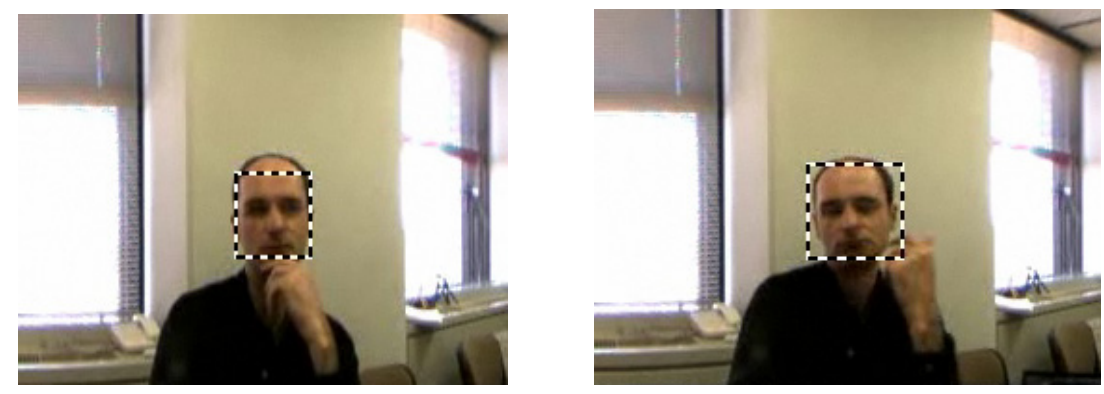

Fig. 2. Front view of the interlocutor shrugging his shoulders

\subsection{Interview and Questionnaire}

The interview study was conducted in international contexts at two locations, Japan and Sweden. In Japan, the subjects were university students and residents at a residence hall for foreign students and scholars in Kyoto, and the test took place in a quiet corner of the common lounge. The 14 subjects were mostly exchange students who had come to Japan to study their particular discipline, and for many, this was their first experience of living abroad. Half of them were just over 20 years of age (7 subjects out of 14), but two older scholars also took part in the experiment. A summary of the subjects' nationality and background is given in Table 1.

In Sweden the subjects were students in an English language communication program at the University of Gothenburg, and the test was part of their course assignment. The subjects were immigrants, exchange students, and native Swedish students, and their experience abroad varied. There were 27 subjects in their 20's, and they represented a wide variety of nationalities. The nationality distribution is given in Table 2.

Considering the subjects' nationality and experience abroad, we tried to select the participants so that there would be subjects from both Eastern and Western cultures, and the numbers are rather equal considering European vs. Asian participants in 
Table 1. Background information of the subjects $(\mathrm{N}=14)$ in Japan. The numbers after the country name indicate the years the person has lived abroad.

\begin{tabular}{llll}
\hline Nationality & Experience abroad & Age group & Gender \\
\hline Catalan & No & $20-24$ & $\mathrm{~F}$ \\
Danish & Belgium (20), Japan (1) & $20-24$ & $\mathrm{M}$ \\
Swedish & US (2), France (1), Spain, Uganda, & $25-44$ & $\mathrm{M}$ \\
& Brazil & & \\
Canadian & US, France, Japan & $45-65$ & $\mathrm{M}$ \\
Moroccan & No & $25-44$ & $\mathrm{~F}$ \\
Uzbek & No & $20-24$ & $\mathrm{~F}$ \\
Filipino & US (4) & $20-24$ & $\mathrm{~F}$ \\
Filipino & US (10) & $45-65$ & $\mathrm{~F}$ \\
Korean & No & $20-24$ & $\mathrm{M}$ \\
Chinese & No & $20-24$ & $\mathrm{M}$ \\
Chinese & No (English teacher) & $25-44$ & $\mathrm{~F}$ \\
Japanese & US (1) & $25-44$ & $\mathrm{~F}$ \\
Japanese & No (work with foreigners) & $25-44$ & $\mathrm{~F}$ \\
Japanese & No & $20-24$ & $\mathrm{M}$ \\
\hline
\end{tabular}

general. However, as the aim was to explore and verify the previously presented hypothesis of the differences in interpretation between Eastern and Western cultures rather than to conduct quantitative study of the interpretation of the shoulder shrug, there was no rigorous effort to get a statistically balances sample of subjects.

Moreover, the setup of the experiment also supported subjective case studies rather than quantitative experiments. The interviews aimed at collecting data of the subjects' own view of the video clip: how they would interpret the particular gesturing and how they would express hesitation themselves. In the multicultural context of exchange students and researchers, the subjects are of course already used to very international communication, and their own cultural context is in the background. Although the interviews of the video clip were not conducted in void, they were conducted in the context which allowed the subjects' interpretation not only of the hesitation and uncertainty presented in the video clip but also of how hesitation and uncertainty are expressed in their own culture, their views being filtered through their own exposure and readiness to admit various differences in intercultural communication.

The setup of the interview study was fairly straightforward. The purpose of the study was first explained to the subjects together with some background of the cultural differences in the interpretation of gestures. In most cases this prompted enthusiastic presentation of the subjects' own experiences and observations of the cultural differences that they have noticed in their own interactions. The subjects were then shown the video clip and they were encouraged to express their observations and opinions of the clip. If they had not noticed the particular shoulder shrug, their attention was drawn to it by verbally describing what the person does. They could see the video as many times as they wanted, and usually 2-3 was enough. 
Table 2. Nationality distribution among the participants $(\mathrm{N}=27)$ in Sweden

\begin{tabular}{cc}
\hline Nationality & Number \\
\hline British & 1 \\
British/Mexican & 1 \\
Cameroonian & 1 \\
Chinese & 6 \\
German & 1 \\
Greek & 1 \\
Iranian & 3 \\
Iraqi & 1 \\
Lithuanian & 1 \\
Pakistani & 1 \\
Romanian & 1 \\
Russian & 3 \\
Swedish & 5 \\
Vietnamese & 1 \\
\hline
\end{tabular}

The subjects were also given a short questionnaire which included questions about their cultural background and interpretation of the video clip. The questionnaire is in the appendix of the paper. Some open-ended questions were aimed at eliciting information about the participants' own interpretation of the event, while the selection of the adjectives was aimed at directing the participants' attention towards the assumed interpretation related to hesitance and uncertainty. The adjectives to describe the gesture and body movement were picked from the Roget's Thesaurus as synonyms and antonyms for the words hesitant and unsure, and then mixed. The selection also included some less frequent words.

\section{Results and Discussion}

\subsection{General Observations}

The analysis of the data collected in Japan confirmed the observation that Chinese and Japanese participants do not use shoulder shrug as a sign of hesitation, and would rather shake their head or move hands sideways. The interpretation of the video clip was described as the speaker being doubtful or puzzled, uncertain, and unsure, and one male participant expressed the speaker's thoughts as "I don't think you are right but I don't want to tell you you're wrong. I don't agree with you". The Japanese subjects also recognized the shoulder shrug as hesitant and unsure, but noted that in Japanese culture, hesitation would normally be expressed verbally or by tilting the head sideways. The Korean male student recognized the gesture as uncertain, unsure, and impolite, but he also said he would use the gesture himself in cases when he was not sure of the situation or did not know the topic. It is interesting that the Swedish participant who was studying in Japan and had lived in various other countries for a 
number of years, would also have expected eye-contact in the situation, so that for him the body movement did not appear only as hesitant, doubtful, reserved, and reluctant, but also impolite. For the Danish subject (who grew up in Belgium), the gesturing conveyed feeling of a reserved, shy, and timid person, who was polite and did not want to impose his views on the others. For the two Filipinos, who had long experience in living in the US, the gesture indicated, somewhat surprisingly, communication that is certain, sure, strong, and confident. They would use similar gesturing if confident and of control of the situation, but would also use more relaxed signaling with smiling and not having hands on chin. The female participants from Morocco, Catalonia, and Uzbekistan all considered the gesture fairly common, and interpreted it as uncertain, irresolute, unsure, indecisive, perplexed, and baffled. The Uzbek would also use it herself if not sure or could not decide what to do, while the Catalan and Moroccan pointed out that the gesture would also convey meaning of "doesn't matter", "as you want", "probably". The young Moroccan lady also noted that the unfinished sentence conveys the meaning that the speaker is not very sure of the idea he's trying to convey, but that the gesture also signals that the person does not care, and this is the way in which she would herself use the gesture.

Concerning the data collected in Sweden, an interesting tendency was that the four Middle Eastern subjects (from Iran and Iraq), seem to interpret the gesture as a sign of a sure, certain and confident speaker, like the Filipinos in the data collected in Japan. One of the subjects wrote that the person on the video is certain about the topic and shows certainty with a friendly gesture, while another wrote that the person tries to emphasize what he is saying. These subjects would not use the gesture themselves because it is regarded impolite and also shows lack of respect for older people, i.e. the gesture is related to behaviour that is socially inacceptable. It is interesting how this interpretation differs from that by the Japanese subjects: now the gesture is used but it expresses inacceptable behaviour unlike in Japan where the gesture is not used at all.

The selection of opposite adjectives to describe the gesture may of course be due to a confusion among the subjects concerning the meaning of the adjectives: e.g. one subject had first marked the "confident" words in the questionnaire but changed them to the "diffident" ones after realizing inconsistency in her markings and explanations. However, it may also be related to the cultural tendency of interpreting the shoulder shrug as conveying the speaker's self-confidence and certainty.

In fact, it is possible that that shoulder shrugging has different underlying interpretations, and "lack of knowledge" associated with it can be interpreted in different ways in different cultures. It can be interpreted as "it is not necessary to say any more" or, as the Moroccan lady noted, "I don't care", both of which can also be associated with confidence and certainty. In this way, a shoulder shrug is understood as a sign of non-continuation which can be associated with both lack of ability to continue and lack of willingness to continue, and thus with uncertainty and confidence/certainty (no need to continue), respectively. Perhaps in some cultures the uncertainty interpretation is primarily focused, while in others the confidence interpretation is primarily focused. Cultural conventions and the use of gestures in general would certainly require more investigations here. 


\subsection{Descriptive Adjectives}

Concerning the word questionnaire, global frequencies of the answers of all the subjects are given in Table 3. As can be seen, the most common way to describe the gesture is to associate it with the adjectives unsure and uncertain (more than half of the subjects), and with doubtful and lack of confidence (about one third of the subjects). There is a tendency to describe the gesture as expressing the speaker's hesitation and lack of knowledge, but it is worth noticing that the adjective hesitant itself does not have especially high frequency ( 10 out of 42 subjects). On the other hand, the gesture is also related to the speaker being reserved or unconvinced (9 out of 42 subjects) or perplexed (8/42). Also the adjectives ambivalent, indecisive, irresolute, or reluctant were picked fairly often (7 out of 42), and the "confidence" adjectives sure and confident have the same frequency (7/42). The latter can be understood as supporting the above hypothesis that the interpretation of the gesture can focus on both the speaker's willingness to continue the conversation and the speaker's ability to say something: $7(16 \%)$ of the subjects interpreted the gesture as expressing the speaker's confidence rather than uncertainty.

We should bear in mind, however, that the selection of appropriate words from the list of descriptive adjectives may be affected by the subjects' knowledge of English. Most of the subjects were non-native speakers, and since their English ability was not tested, the word-test need not accurately reflect their understanding of the situation.

Table 3. Global frequencies $(\mathrm{N}=42)$

\begin{tabular}{|c|c|c|c|c|}
\hline $\begin{array}{c}\text { hesitant } \\
\mathbf{1 0}\end{array}$ & unhesitant & troubled & doubtful & distracted \\
\hline confused & disturbed & puzzled & certain & uncertain \\
5 & 3 & 6 & $\mathbf{6}$ & $\mathbf{2 3}$ \\
\hline resolute & irresolute & perplexed & reserved & unconvinced \\
1 & 7 & $\mathbf{8}$ & $\mathbf{9}$ & $\mathbf{9}$ \\
\hline strong & wavering & weak & shy & incoherent \\
3 & 5 & 2 & 4 & 6 \\
\hline timid & bold & bashful & confused & unwavering \\
2 & 1 & 2 & 4 & \\
\hline reluctant & sorry & afraid & baffled & ambivalent \\
7 & & 1 & 4 & 7 \\
\hline sure & unsure & decisive & confident & indecisive \\
$\mathbf{7}$ & $\mathbf{2 2}$ & & $\mathbf{7}$ & 7 \\
\hline polite & impolite & apologetic & regretful & inconfident* \\
4 & 3 & & 1 & $\mathbf{1 2}$ \\
\hline
\end{tabular}


However, besides few markings on the other adjectives, the general tendency comes clear in the word test: the gesture is described as expressing the speaker's uncertainty and lack of knowledge. Further more, most subjects interpret the gesture as signalling the speaker is indecisive about what to say (hesitant about the topic), but $16 \%$ interpret the speaker's disinclination to speak as the speaker being sure and certain in the situation (no need or willingness to continue).

Typically the subjects selected 4-5 adjectives, except for the Greek and Uzbek, who had picked the total of 13 and 10 adjectives, respectively. Based on the selected adjectives, and a few background features of the subjects such as age, nationality, experience abroad, we then formed vectors that characterized each subject concerning their interpretation of the shrug, and then used these vectors to compare the interpretations automatically. Comparison was done with the Support Vector Machine technique in the Weka software [11], as a simple binary classification of the vectors into those that represented Western (European) vs. Eastern (non-European) culture groups. The classification reached $75 \%$ accuracy over 10 -fold cross-validation, i.e. $3 / 4$ of the subjects were correctly classified into either group on the basis of the features encoded in the vectors.

Considering then how similar the vector representations are, and if they form any natural clusters on the basis of the adjectives, we used the Expectation-Maximization clustering of Weka. The evaluation of the clusters with respect to the two culture groups produced $61 \%$ accuracy, i.e. almost $2 / 3$ of the subjects had similar characterizing vectors (had selected similar adjectives) as those who belong to the same culture group.

Both accuracies can be considered fair results, especially as the dataset is small and the vectors are sparse. However, as already mentioned, our study was not intended as a statistical analysis of the interpretation of the shoulder shrug but as a preliminary exploration of intercultural communication. It is obvious that a larger group of subjects with a balanced sampling of various affecting characteristics and parameters would be necessary for a more detailed statistical study, but it is interesting that this kind of preliminary classification and clustering experiments already provide support for the cultural differences and the tendency to describe the shoulder shrug differently in the Eastern and Western cultures is visible in the answers drawn from a small set of 42 individuals only.

Finally, the word selection test also showed that a more detailed analysis of the semantics of hesitation related words is necessary. In the above experiments, using Weka's automatic feature analysis techniques, we found that the adjectives certain and unconvinced as well as unhesitant and inconfident were selected as the most predictive adjectives with respect to the two classes (culture groups). The selection was based solely on the statistical properties of the vectors by measuring the information gain with respect to the class, and considering the individual predictive ability of each adjective together with the degree of redundancy between them (i.e. correlation of the adjectives with the classes). The result is in accordance with what has been discussed above qualitatively: interpretation of the shoulder shrug can be described in terms of Western (European) view of the speaker who is unconvinced and inconfident or with the Eastern (non-European) view of a certain and unhesitant speaker (which further implies that the gesture is not much used as too arrogant behaviour is socially inacceptable). However, it would be useful to conduct a 
linguistic analysis of the words describing hesitation related phenomena and to cluster their occurrence according to their semantic similarity and the context in which they occur. This will be future work.

\subsection{Speech Analysis}

Hesitation marking in speech is fairly varied. In English this ranges from different types of hesitation markers (uhh, umm) to pauses (silence) and slow speaking rate. Fundamental frequency F0 is also shown to rise before pauses occurring in major syntactic boundaries, but not if the pause occurs elsewhere. In Japanese, [10] found that the prosodic and temporal features of a response carry information about how the speaker has grounded the information expressed in the partner's previous utterance, especially if the speaker repeats a portion of that utterance. The features such as longer delays, higher pitch, slower tempo, and rising boundary tone signal lower integration degree, i.e. the information is not fully integrated in the speaker's body of knowledge, and in the context of this paper we can say that they express hesitation and uncertainty.

Figure 3 (a screenshot by the Praat software, http://www.fon.hum.uva.nl/praat/) shows speech analysis of the end part of the speaker's utterance it's just a... which occurs immediately before a longer silence and the shoulder shrug. In the screenshot, red points show variation of the first format F0, the blue line shows the pitch contour, and the green continuous line shows intensity.

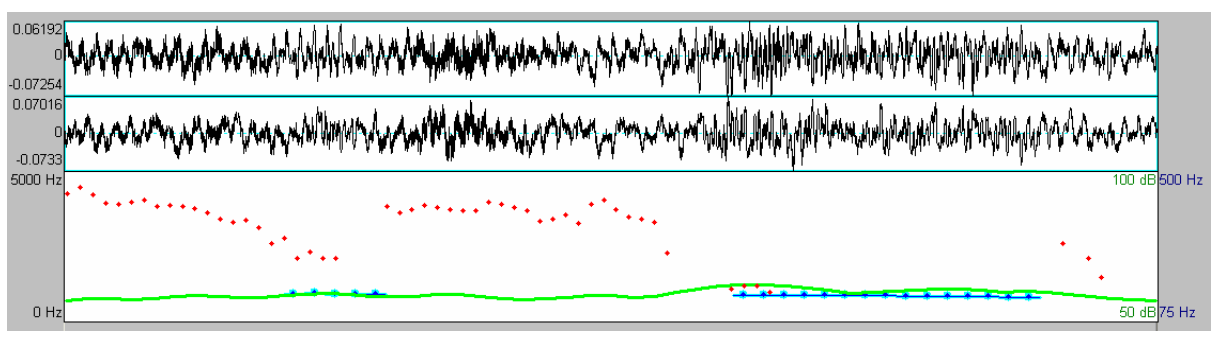

Fig. 3. Combined speech analysis in Praat (see text)

It can be seen that the utterance conforms to all the typical prosodic characteristics related to hesitations, and does not differ from the speaker's other hesitative utterances either. The speaker does not raise his voice (mean energy is about $60 \mathrm{~dB}$ ), but lengthens the function word so that the speaking rate seems to slow down. The pitch contour stays flat, and shows no upward tendency immediately before the pause (and the shoulder shrug), which is expected since the pause does not occur at a major syntactic boundary.

Considering the correlation of prosody and hesitation related communicative body movements, an interesting question is thus if the two modalities, speech and gesturing support each other or if they operate independently of in the communicative situation. It seems that the shoulder shrugging gesture and prosodic marking have no special correlation in this particular case: the shrug occurs during the pause and it seems more natural to relate the prosody to the unfinished utterance and uncertainty in general, 
rather than to the particular gesture. Following this line of thought, it seems safe to assume that the gesture is used to emphasize the speaker's uncertainty, or to add "don't care" -type meaning association - as some subjects described the gesture. In fact, it may be justified to conclude that the shoulder shrug in this particular case has a status of an independent communicative act itself, i.e. after the unfinished utterance it's just $a$, the speaker continues with a shoulder shrug to finish off the sentence with a gesture expression in order to indicate that it is not important to express, or that he is not able to express the actual content in words.

\section{Conclusions and Future Work}

The current work is based on an interview study of subjects with different cultural background, concerning their interpretation of a particular shoulder shrugging. We confirmed the previous hypothesis of the distinction between Eastern and Western cultures, and the differences in interpreting and using shrugging gestures. We also found out that in Middle-Eastern cultures the same gesturing tends to be interpreted in an opposite way: as a sign of a confident and certain interlocutor. Because this kind of behaviour may also imply arrogance, the gesture can also be considered socially inacceptable and impolite. As a result, we concluded that the underlying interpretation concerning shoulder shrugging may be related to "lack of knowledge" which can then be further analysed in terms of "lack of ability to continue" and "lack of willingness to continue", and that different cultures then may focus more on the uncertainty interpretation while the others focus more on the confidence interpretation.

However, in order to draw statistical conclusions of intercultural communication strategies and to make generalizations about hesitation related expressions, especially shoulder shrugging, in wider cultural contexts, it is important to collect more data on the hesitation expressions in different cultures, and also more examples of shoulder shrugging itself. We can then use a similar kind of methodology as applied in the current work, to collect information about the appropriate interpretations of these expressions. Interviews of people with different cultural background would allow us to collect data on the participants' subjective views and interpretations of the selected video examples, while a word test -type questionnaire would provide us with more objective information about non-verbal signals and their functions in communication. This way we can study how hesitation, uncertainty, doubt, and lack of knowledge are expressed in different cultures, and also how particular expressions, such as shoulder shrugs, are interpreted in different cultural contexts.

We can also include activity analysis into the study and observations of uncertainty in various types of social activities, and consequently, obtain further insights about different manifestations of hesitation related phenomena. The roles of the interlocutors in different activities support different behaviour patterns and thus also differences in the acceptable ways of expressing one's hesitation and ignorance. For instance, communication strategies used when talking to one's superior or a colleague or when chatting with a familiar or an unfamiliar partner, are likely to differ from each other concerning the type and frequency of expressions, the use of gesturing and body movements, and tolerance of silence. Moreover, the five dimensions of [5], i.e. hierarchy, individualism, gender, uncertainty, and orientation, can also be associated 
with the roles and activities that the individuals in different cultures are involved in, and consequently, cultural differences can be studied from point of view of social activities that the individuals as members of various social groups take part in. Intercultural communication can thus be analysed in terms of social activities and interactions among individual agents, instead of contrasting stereotypical behaviours.

In ICT (Intercultural Communication Technology) virtual humans and embodied communicative agents form an important application area and the behaviour of such artificial agents is becoming more human-like. Such applications aim at recognizing and monitoring the user's behaviour - both verbal and non-verbal - and providing responses that are appropriate given the task (e.g. factual information exchange) and the interaction context. As the context also includes cultural context, adaptation of the agents to different languages and cultures is a relevant topic that needs to be modelled as well. For instance, in educational applications, training environments, and virtual companions such culture-specific factors affect the users' learning and enjoyment, and taking them into consideration can improve the efficiency and effectiveness of the applications. In these cases, the starting point has usually been an explicit indication of the level of understanding by the user, and it has been common to study conversational feedback and grounding processes in order to provide the users with appropriate responses and effective help in problematic situations. This kind of constructive feedback is useful if we consider the participants' understanding and interpretation, i.e. the intake of the information and its grounding in the existing background information. Hesitation related information is also important in this context since it allows the interlocutors to express the degree of understanding and the degree of commitment to the presented topic. In the smooth communication it is important to convey agreement and emotional stance to the partner, i.e. besides their cognitive understanding of the information, the speakers should also indicate their degree of commitment to the presented information. Studies of various hesitation related phenomena can therefore complement the grounding analysis in the analysis and construction of shared understanding and mutual bonds.

Acknowledgements. The first author thanks NICT and Doshisha University for the opportunity to collect the interview data and conduct the first analyses while a NICT Visiting Fellow at Doshisha University, Kyoto.

\section{References}

1. Allwood, J.: Tvärkulturell kommunikation in Allwood, J (Ed.) Tvärkulturell kommunikation, Papers in Anthropological Linguistics 12, University of Göteborg, Dept of Linguistics. Intercultural Communication, Also in English (1985)

2. Allwood, J.: Bodily Communication - Dimensions of Expression and Content. In: Granström, B., House, D., Karlsson, I. (eds.) Multimodality in Language and Speech Systems, pp. 7-26. Kluwer Academic Publishers, Dordrecht (2002)

3. Endrass, B., Rehm, M., André, E.: Culture-specific Communication Management for Virtual Agents. In: Proceedings of the 8th International Conference on Autonomous Agents and Multiagent Systems (AAMAS), Budapest, Hungary, pp. 281-288 (2009)

4. Hall, E.T.: Beyond Culture. Anchro Press, Garden City NY (1976)

5. Hofstede, G.: Culture's consequences: International differences in work-related values. Sage, Newbury Park (1980) 
6. Jan, D., Herrera, D., Martinovski, B., Novick, D., Traum, D.: A computational Model of Culture-specific Conversational Behavior. In: Proceedings of Intelligent Virtual Agents Conference, pp. 45-56 (2007)

7. Jokinen, K., Campbell, N.: Non-verbal Information Sources for Constructive Dialogue Management. In: Tutorial at LREC-2008, Marrakech, Morocco (2008)

8. Jokinen, K., Wilcock, G.: Contextual Inferences in Intercultural Communication. In: Arppe, A., et al. (eds.) A Man of Measure. Festschrift in the Honour of Fred Karlsson. Finnish Linguistic Association, pp. 289-298 (2005)

9. Pajupuu, H.: Cultural Context, Dialogue, Time. Estonian Academy of Sciences, Institute of the Estonian Language, Tallinn (1995)

10. Shimojima, A., Katagiri, Y., Koiso, H., Swerts, M.: An Experimental Study on the Informational and Grounding Functions of Prosodic Features of Japanese Echoic Responses. In: The Proceedings of ESCA Workshop on Dialogue and Prosody, pp. 187192 (1999)

11. Witten, I.H., Frank, E.: Data Mining:Practical machine learning tools and techniques, 2nd edn. Morgan Kaufmann, San Francisco (2005)

\section{Appendix}

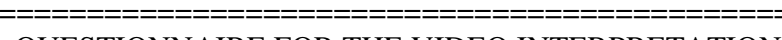 \\ QUESTIONNAIRE FOR THE VIDEO INTERPRETATION}

Please fill in the form and return it to the instructor.

AGE:

GENDER:

MOTHER TONGUE:

WHICH OTHER LANGUAGES DO YOU SPEAK?

Please indicate also the level of your knowledge:
(1) like native
(2) good command
(3) sufficient (for daily life)
(4) basic knowledge only

NATIONALITY:

WHICH COUNTRIES HAVE YOU LIVED IN AND FOR HOW LONG? 
HOW WOULD YOU INTERPRET THE PERSON'S BODY MOVEMENT

IN THE VIDEO?

Choose as many alternatives as you like:

\begin{tabular}{|c|c|c|c|c|}
\hline hesitant ___ & unhesitant & troubled ___ & doubtful __ & distracted \\
\hline confused ___ & disturbed & puzzled ___ & certain ___ & uncertain \\
\hline resolute & irresolute & perplexed & reserved & unconvinced \\
\hline strong & wavering ___ & weak & shy __ & incoherent \\
\hline timid ___ & bold ___ bashful & _ confused & - unv & ing $\ldots$ \\
\hline reluctant & sorry ___ & afraid & baffled __ & ambivalent \\
\hline sure & unsure & decisive & confident & indecisive \\
\hline polite & impolite & apologetic & regretful & inconfident \\
\hline
\end{tabular}

PLEASE DESCRIBE THE MEANING OF THE BODY MOVEMENT IN YOUR OWN WORDS:

WOULD YOU USE SIMILAR GESTURING IN A SIMILAR SITUATION YOURSELF?

YES

$\mathrm{NO}$

IF YES, WHY?

IS IT COMMON IN YOUR CULTURE?

IF NOT, WHY NOT?

WHAT KIND OF GESTURING WOULD YOU USE IN A SIMILAR SITUATION IN YOUR CULTURE? 
THANK YOU VERY MUCH FOR YOUR TIME! WE APPRECIATE YOUR HELP!

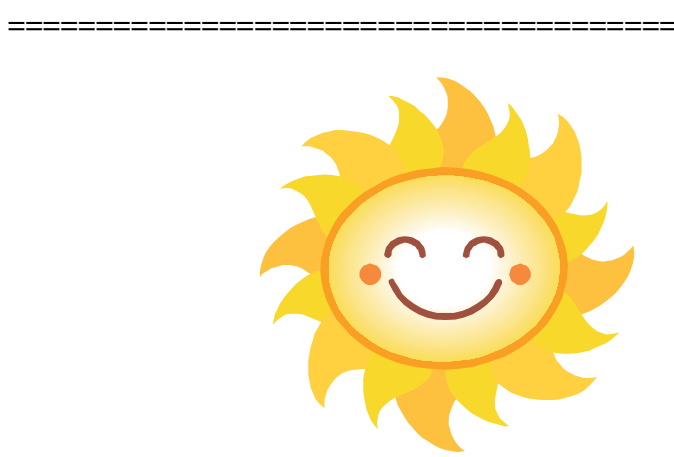

